\title{
Lot or Batch Number Production Identifier
}

National Cancer Institute

\section{Source}

National Cancer Institute. Lot or Batch Number Production Identifier. NCI Thesaurus.

Code C101672.

The production identifier is the lot or batch number. 